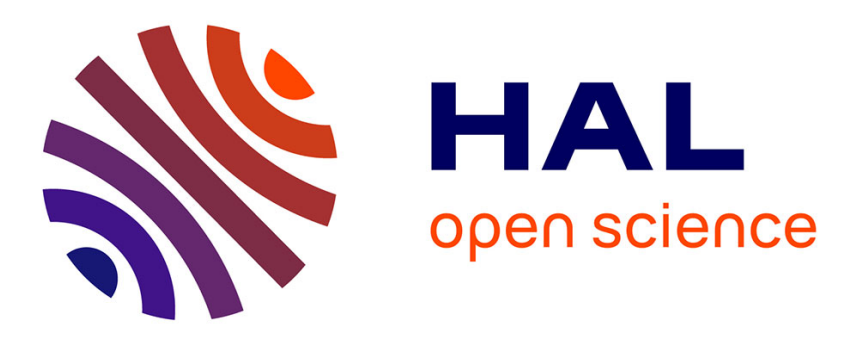

\title{
Adaptive filtering for robust proprioceptive robot impact detection under model uncertainties
}

Maria Makarov, Alex Caldas, Mathieu Grossard, Pedro Rodriguez-Ayerbe, Didier Dumur

\section{- To cite this version:}

Maria Makarov, Alex Caldas, Mathieu Grossard, Pedro Rodriguez-Ayerbe, Didier Dumur. Adaptive filtering for robust proprioceptive robot impact detection under model uncertainties. IEEE/ASME Transactions on Mechatronics, 2014, 19 (6), pp.1917-1928. 10.1109/TMECH.2014.2315440 . hal00998960

\section{HAL Id: hal-00998960 \\ https://hal-centralesupelec.archives-ouvertes.fr/hal-00998960}

Submitted on 2 Nov 2021

HAL is a multi-disciplinary open access archive for the deposit and dissemination of scientific research documents, whether they are published or not. The documents may come from teaching and research institutions in France or abroad, or from public or private research centers.
L'archive ouverte pluridisciplinaire HAL, est destinée au dépôt et à la diffusion de documents scientifiques de niveau recherche, publiés ou non, émanant des établissements d'enseignement et de recherche français ou étrangers, des laboratoires publics ou privés. 


\title{
Adaptive Filtering for Robust Proprioceptive Robot Impact Detection under Model Uncertainties
}

\author{
M. Makarov, A. Caldas, M. Grossard, P. Rodriguez-Ayerbe and D. Dumur
}

\begin{abstract}
In the context of safe human-robot physical interaction, the present article introduces a new method for the detection of dynamic impacts of flexible-joint robot manipulators with their environment. The objective is to detect external impacts applied to the robot using only proprioceptive information with maximal sensitivity. Several model-based detection methods in robotics are based on the difference, called residual, between the estimated and the actual applied torques. Sensitivity of such methods can be limited by model uncertainties that originate either from errors on experimentally identified model parameters, possibly varying with the operating conditions, or the use of simplified models, which results in a residual dependence on the robot's state. The main contribution of this paper consists of a new adaptive residual evaluation method that takes into account this dependence, which otherwise can lead to a trade-off between sensitivity and false alarm rate. The proposed approach uses only proprioceptive motor-side measurements and does not require any additional joint position sensors or force/torque sensors. Dynamic effects of a collision on the residual are isolated using band-pass filtering and comparison with a state-dependent dynamic threshold. Adaptive on-line estimation of filter coefficients avoids the need for extensive experiments for parametric model identification. Experimental evaluation on the CEA backdrivable ASSIST robot arm illustrates the enhancement of the detection sensitivity.
\end{abstract}

Index Terms-adaptive filters, fault detection, human-robot interaction, manipulator dynamics, uncertainty.

\section{INTRODUCTION}

$\mathbf{S}$ AFETY is a major concern in robotic industrial or service applications where humans and robot manipulators share the same workspace. To realize safe robot systems in unstructured environments, and minimize injury risks related to collisions between the human operator and moving parts of the robot, both preventive and reactive effective approaches are required. Among preventive approaches, supervision systems based on 3D simulation and exteroceptive sensors can be used to locate the robot with respect to its environment, and avoid collisions by appropriate path planning and human monitoring [1]-[4]. In a complementary way, reactive control strategies aim at minimizing the effects of unexpected collisions, in which case the main injury risk factors depend on the trajectory characteristics (e.g. speed), the robot's intrinsic properties as inertia and rigidity, as well as its ability to rapidly detect external forces from proprioceptive measurements only. Meth-

M. Makarov, P. Rodriguez-Ayerbe and D. Dumur are with SUPELEC Systems Sciences (E3S), Control Department, F-91192 Gif sur Yvette, France.

A. Caldas and M. Grossard are with CEA, LIST, Interactive Robotics Laboratory, Gif sur Yvette, F-91190, France.

Corresponding author: M. Makarov (e-mail: maria.makarov@supelec.fr phone: +331698513 75). ods to distinguish between an intended contact and unexpected collisions were also proposed in [5].

In this context, lightweight manipulators provide interesting intrinsic properties in terms of inertia and flexibility in comparison with heavier manufacturing robots, and are also designed to develop lower maximal torques, which are less likely to lead to fatal injuries [6]. Other risk assessment criteria are compression force, impact force and pressure per surface exerted by the robot in case of an impact [7]. Joint stiffness was experimentally shown in [8] to contribute to human safety, according to a criterion based on clamping and impact force. The criterion of somatic pain has been experimentally investigated in [9], [10], where thresholds of maximum tolerated contact forces were proposed. With regard to the previously cited criteria, any contact forces in case of an unexpected collision must be detected with maximal sensitivity using proprioceptive information only. A first approach consists of achieving this requirement through extended sensing capabilities (motor and joint position sensors, force/torque sensors at joint level [11] or at base and wrist [12]) allowing for efficient interaction schemes, as in [13] where Cartesian impedance control combined with online path planning depending on the estimated external force was applied to a lightweight robot equipped with joint torque sensors. A second approach relies on traditional motor-side measurements only, which represents a realistic situation for a large class of industrial robots under integration and cost constraints. In this latter case, the backdrivability level of the robot's transmissions determines the maximum detection sensitivity that can be achieved. Indeed, external efforts applied to the robot can only be detected through their reflection on the motor shafts. The more backdrivable the mechanical structure is, the more sensitive the detection can possibly be. In the present paper, detection algorithms are investigated on the example of adapted mechanical structures specifically designed for human-robot interaction, such as backdrivable manipulators with intrinsically low friction levels [14] in which external forces are reflected with high fidelity at the motor level without the need of additional joint torque sensors.

The detection of collisions between the robot and its environment without additional force sensors can be seen as a special case of the fault detection and isolation problem. Non model-based methods present the advantage of being independent of a specific model, usually relying on direct characteristics of signals involved in control as amplitude or instantaneous variation of position error or control signal [15]. These methods directly depend on the structure, type and gains of the controllers used, and therefore can not be considered 
in a separate way from the system control architecture. The present paper considers model-based methods, which rely primarily on the system model, and can be designed more independently from the system tracking performance. Modelbased fault detection strategies generally comprise two main steps, namely the generation of a residual signal carrying the fault signature, and the residual evaluation enabling the detection and the production of a decision (Fig. 1).

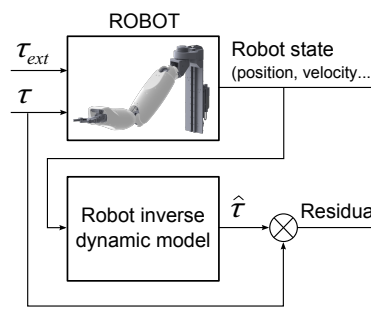

Step 1: Residual generation

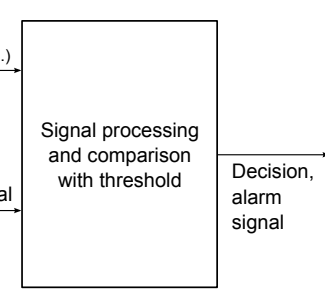

Step 2: Residual evaluation

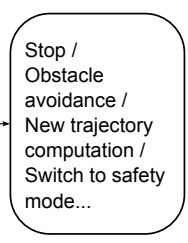

Step 3: Post-impact strategy
Fig. 1. Main steps of a collision detection algorithm, with $\tau_{\text {ext }}$ the collision torque vector and $\hat{\tau}$ the motor torque estimate.

For the first step, various residual generation methods are provided by control theory, since faults in dynamic systems generally result in variations of state variables or model parameters. Quantitative model-based diagnostic methods therefore rely on state observers or on-line parameter estimation techniques [16]-[19]. A method based on a state observer with nonlinear friction compensation for detection of external torques applied to an industrial robot is proposed in [20]. In robotics, residuals can also be generated using the inverse dynamic model as the difference between the applied and the estimated motor torque, which can be easily computed in real time using only motor-side informations in case of rigid robots. An enhancement of this approach is based on the generalized momentum of the robot, and does not require the computation of the acceleration and thus reduces the measurement noise influence [11], [21]. Another method based on torque filtering that does not require any acceleration measures or estimates is presented in [22]. In a similar perspective, the benefits of band-pass filtering of joint torque for robust collision detection were highlighted in [23].

Regardless of the residual generation method, the difficulty in realizing detection algorithms that would be sensitive for a large variety of operating conditions resides in the fact that modeling errors affect the residual in the same structural way as external disturbances. Variations in model parameters due to varying load or friction dependence on the temperature are examples of such uncertainties. A good residual evaluation method therefore must distinguish the effects of modeling errors on the residual from those of a real collision. A basic evaluation method consisting in a comparison with a static threshold can be sufficient in case where the model presents few uncertainties. However, in the opposite case, it usually leads to a trade-off between sensitivity and false alarm rate (Fig. 2), with a risk of excessively conservative thresholds fixed to values greater than the maximum model error for a given trajectory.

In order to overcome this difficulty, two different approaches

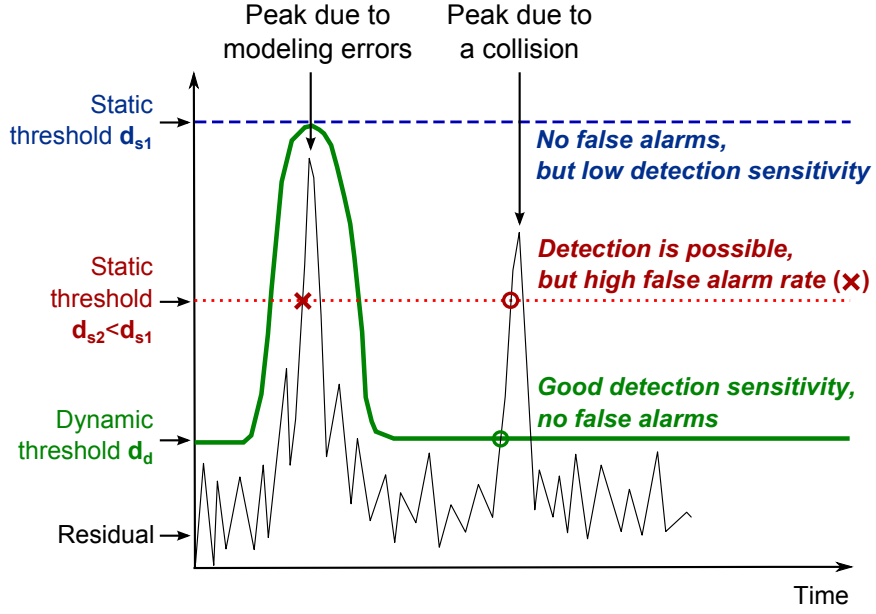

Fig. 2. Static versus dynamic threshold in residual evaluation.

have been described in the literature. A dynamic threshold is defined in [20] to represent the residual dependence on the state of the robot (position, velocity, acceleration) using fuzzy logic rules. This first approach may require a large number of experiments to formulate and test the logic rules in an exhaustive way for the whole robot workspace. Other authors envisage on the contrary an on-line estimation of the model parameters, applied mainly to the rigid robot case, and based on the linearity of the robot dynamic model with respect to its parameters. In this second approach, model parameters are estimated either separately or simultaneously to the detection phase. Adaptation is performed on a collision free trajectory prior to the actual detection phase in [22], which means that a new adaptation phase is required at every change in the reference trajectory. Simultaneous adaptation and detection is presented in [24] for a robot in interaction with its environment using an adaptive impedance control law. In case of a position controlled robot, it is achieved in [25] for actuator fault detection and isolation by means of an overparametrization of the rigid dynamic model. Such an approach takes into account parameter variations within a fixed rigid model structure, and therefore may not allow to represent other types of uncertainties.

The present article describes a novel collision detection algorithm for flexible-joint robots, based on the analysis of the residual dynamics in presence of uncertainties, leading to a new residual evaluation method which aims at separating the dynamic effects of a collision from the effects of modeling errors. The presented method does not require additional sensors and therefore is of particular interest in an industrial context of reduced measurements, when only motorside position and current information is available. A standard rigid inverse dynamic model is used to compute the residual, without the need of extensive identification experiments of a specific refined model. A simplified structure for the model of the residual is first deduced from its physical dynamic model. Based on this model, the residual is high-pass filtered and compared with a state-dependent dynamic threshold computed as output of a multivariable filter. Without the need of extensive identification experiments, filter coefficients are adapted 
on-line in a recursive least squares method to account for deviations from the initial rigid model. The proposed method provides high-level tuning parameters which can be easily adjusted.

Section II is devoted to the derivation of the residual dynamics under modeling uncertainties. The consequences of parametric uncertainties and those of neglecting transmission flexibilities in the residual computation are emphasized and experimentally illustrated. Section III describes the proposed method which relies on a residual model structure motivated by the previously derived dynamics. Appropriate residual filtering and a dynamic threshold are defined, the adaptive formulation of the proposed algorithm is given, and details are provided on the elaboration of the detection signal from a comparison of the filtered residual with the dynamic threshold. In Section IV, experiments illustrate the benefits of the proposed method with respect to a static threshold, and an evaluation of the detection sensitivity is provided using an external force sensor.

\section{DETECTION UNDER UNCERTAINTIES - SYSTEM MODELING}

\section{A. Robot dynamic models}

The dynamic behavior of serial robot manipulators can be generally represented by three different types of models, depending on the physical structure and phenomena that need to be emphasized: $i$ ) rigid links and rigid joints, $i i)$ rigid links and flexible joints, iii) flexible links [26]. The rigid link/flexible-joint model, taking into account the transmission flexibilities that can arise in the actuation chain, is well suited to represent the behavior of lightweight robots designed for interaction. For a $n$-dof serial robot, let $\mathbf{x}_{m} \in \mathbb{R}^{n}$ and $\boldsymbol{\tau}_{m} \in \mathbb{R}^{n}$ denote the motor positions and torques, $\mathbf{R}_{\text {red }}$ the transmission matrix between motors and joints regrouping the reduction ratios, $\mathbf{x}_{1} \in \mathbb{R}^{n}$ and $\boldsymbol{\tau} \in \mathbb{R}^{n}$ the motor positions and torques after the reduction stage such that $\mathbf{x}_{1}=\mathbf{R}_{r e d}^{-1} \mathbf{x}_{m}$ and $\boldsymbol{\tau}=\mathbf{R}_{\text {red }} \boldsymbol{\tau}_{m}$, and $\mathbf{x}_{2} \in \mathbb{R}^{n}$ the joint position vector. The reduced flexible-joint model can be expressed as follows [27]:

$$
\begin{aligned}
\mathbf{M}\left(\mathbf{x}_{2}\right) \ddot{\mathbf{x}}_{2}+\mathbf{H}\left(\mathbf{x}_{2}, \dot{\mathbf{x}}_{2}\right)+\boldsymbol{\tau}_{f a}+\mathbf{K}\left(\mathbf{x}_{2}-\mathbf{x}_{1}\right) & =\boldsymbol{\tau}_{e x t} \\
\mathbf{I}_{m o t} \ddot{\mathbf{x}}_{1}+\boldsymbol{\tau}_{f m}-\mathbf{K}\left(\mathbf{x}_{2}-\mathbf{x}_{1}\right) & =\boldsymbol{\tau}
\end{aligned}
$$

with $\mathbf{M}\left(\mathbf{x}_{2}\right) \in \mathbb{R}^{n \times n}$ the robot inertia matrix, $\mathbf{I}_{m o t} \in \mathbb{R}^{n \times n}$ the constant diagonal motor inertia matrix, $\mathbf{K} \in \mathbb{R}^{n \times n}$ the stiffness matrix, $\mathbf{H}\left(\mathbf{x}_{2}, \dot{\mathbf{x}}_{2}\right)=\mathbf{C}\left(\mathbf{x}_{2}, \dot{\mathbf{x}}_{2}\right) \dot{\mathbf{x}}_{2}+\mathbf{g}\left(\mathbf{x}_{2}\right) \in \mathbb{R}^{n}$ regrouping the contributions of gravity $\mathbf{g}\left(\mathbf{x}_{2}\right)$ and Coriolis and centrifugal torques $\mathbf{C}\left(\mathbf{x}_{2}, \dot{\mathbf{x}}_{2}\right) \dot{\mathbf{x}}_{2}$, and $\boldsymbol{\tau}_{f a}, \boldsymbol{\tau}_{f m} \in \mathbb{R}^{n}$ respectively the joint and motor friction torques. Additional damping effects can be included between $\mathbf{x}_{1}$ and $\mathbf{x}_{2}$ in the flexible transmission model. Without loss of generality, they will not be considered in what follows to simplify the analysis. The external torque vector is denoted $\tau_{\text {ext }} \in \mathbb{R}^{n}$, and for the example of an external force $\mathbf{F}_{\text {ext }}$ applied at the end-effector of the robot is given by $\tau_{\text {ext }}=\mathbf{J}\left(\mathbf{x}_{2}\right)^{T} \mathbf{F}_{\text {ext }}$, where $\mathbf{J}$ is the Jacobian matrix.

In the following, we consider that only motor-side measurements (position, current) are available, which represents a realistic assumption for a large class of industrial robots, under integration and cost constraints. In the adopted strategy, the rigid model is directly used to estimate the motor torque in real time, which simplifies on-line computations in comparison to the flexible-joint model. When the joints are considered as perfectly stiff $(\mathbf{K} \rightarrow \infty), \mathbf{x}_{1}=\mathbf{x}_{2}$ and equations (1-2) reduce to the rigid model:

$$
\mathbf{M}_{r i g}\left(\mathbf{x}_{1}\right) \ddot{\mathbf{x}}_{1}+\mathbf{H}\left(\mathbf{x}_{1}, \dot{\mathbf{x}}_{1}\right)+\boldsymbol{\tau}_{f}=\boldsymbol{\tau}+\boldsymbol{\tau}_{\text {ext }}
$$

with $\mathbf{M}_{r i g}\left(\mathbf{x}_{1}\right)=\mathbf{M}\left(x_{1}\right)+\mathbf{I}_{m o t} \in \mathbb{R}^{n \times n}$ the rigid robot inertia matrix, and $\tau_{f}$ the friction torque. In the following, the Coulomb friction model is considered. For the $j$-th joint:

$$
\begin{aligned}
\tau_{f m_{j}} & =F_{v m_{j}} \dot{x}_{1_{j}}+F_{s m_{j}} \operatorname{sign}\left(\dot{x}_{1_{j}}\right) \\
\tau_{f a_{j}} & =F_{v a_{j}} \dot{x}_{2_{j}}+F_{s a_{j}} \operatorname{sign}\left(\dot{x}_{2_{j}}\right)
\end{aligned}
$$

and

$$
\tau_{f_{j}}=F_{v_{j}} \dot{x}_{1_{j}}+F_{s_{j}} \operatorname{sign}\left(\dot{x}_{1_{j}}\right), x_{2_{j}}=x_{1_{j}}
$$

where $\operatorname{sign}(\cdot)$ denotes the sign function, $F_{v_{j}}\left(F_{v m_{j}}, F_{v a_{j}}\right)$ are the viscous friction coefficients (respectively at motor and joint levels), and $F_{s_{j}}\left(F_{v m_{j}}, F_{v a_{j}}\right)$ are the Coulomb friction coefficients (respectively at motor and joint levels).

\section{B. Residual generation based on rigid model}

The residual is defined as the difference between the applied motor torque $\tau$ and the estimated motor torque $\hat{\tau}$ :

$$
\mathbf{r}:=\hat{\tau}-\boldsymbol{\tau}
$$

with $\hat{\tau}$ resulting from the rigid model (3) in the absence of external torques:

$$
\hat{\boldsymbol{\tau}}=\hat{\mathbf{M}}_{\text {rig }}\left(\mathbf{x}_{1}\right) \ddot{\mathbf{x}}_{1}+\hat{\mathbf{H}}\left(\mathbf{x}_{1}, \dot{\mathbf{x}}_{1}\right)+\hat{\boldsymbol{\tau}}_{f}
$$

In case of rigid robots driven by DC motors, the motor torque $\boldsymbol{\tau}$ can be obtained from the motor currents $\mathbf{i}_{m}$ as $\boldsymbol{\tau}=\mathbf{R}_{r e d}^{-1} \mathbf{K}_{e m} \mathbf{i}_{m}$ with $\mathbf{R}_{r e d}$ the reduction matrix and $\mathbf{K}_{e m}$ the diagonal matrix of torque constants of different involved motors. Backdrivable robots with intrinsically low friction levels are especially advantageous in this situation since external forces are reflected with high fidelity on the motor torque without the need of additional joint torque sensors.

In the ideal case of rigid robots $\left(\mathbf{x}_{1}=\mathbf{x}_{2}\right)$ with a perfectly known model, the residual is equal to the external torque $\tau_{\text {ext }}$ applied to the robot:

$$
\mathbf{r} \underset{\text { ideal,rigid }}{=} \boldsymbol{\tau}_{\text {ext }}
$$

Still for rigid robots, taking into account parametric uncertainties on terms $\mathbf{M}_{\text {rig }}\left(\mathbf{x}_{1}\right)$ and $\mathbf{H}\left(\mathbf{x}_{1}, \dot{\mathbf{x}}_{1}\right)$ that affect the torque estimate leads to the following expression of the residual $\mathbf{r}$ :

$$
\mathbf{r}=\boldsymbol{\tau}_{\text {ext }}+\Delta \mathbf{M}_{\text {rig }}\left(\mathrm{x}_{1}\right) \ddot{\mathbf{x}}_{1}+\Delta \mathbf{H}\left(\mathrm{x}_{1}, \dot{\mathbf{x}}_{1}\right)+\boldsymbol{\Delta} \boldsymbol{\tau}
$$

with uncertainties defined by:

$$
\begin{aligned}
& \Delta \mathbf{M}_{r i g}\left(\mathbf{x}_{1}\right)=\hat{\mathbf{M}}_{r i g}\left(\mathbf{x}_{1}\right)-\mathbf{M}_{r i g}\left(\mathbf{x}_{1}\right) \\
& \Delta \mathbf{H}\left(\mathbf{x}_{1}, \dot{\mathbf{x}}_{1}\right)=\hat{\mathbf{H}}\left(\mathbf{x}_{1}, \dot{\mathbf{x}}_{1}\right)-\mathbf{H}\left(\mathbf{x}_{1}, \dot{\mathbf{x}}_{1}\right) \\
& \boldsymbol{\Delta} \boldsymbol{\tau}=\hat{\tau}_{f}-\tau_{f}
\end{aligned}
$$


This expression brings out the dependence, even in the perfectly rigid case, of the residual on the acceleration (inertia uncertainty), speed (friction, Centrifugal and Coriolis uncertainty) and position (gravity uncertainty). In the next section, the dynamics of the residual when the robot is considered to have flexible joints is analyzed.

\section{Residual dynamics for flexible-joint robots}

When the transmission flexibilities are taken into account using the flexible-joint model (1-2), other effects and higher order dynamics can be shown to affect the residual. In the following, the static friction contribution will be considered as an additive disturbance which effects have been already emphasized in (10). Static friction is therefore neglected in the following derivations of the residual dynamics, even if its effects are eventually taken into account in the proposed method.

The flexible-joint robot with motor-side measurements only can be seen as an underactuated system. To analyze the dynamics of the residual defined by (7) in presence of joint flexibilities, its expression is rewritten as a function of motor variables by eliminating the joint variables which are not measured. The dependence in $\mathbf{x}_{2}$ of $\mathbf{M}$ and $\mathbf{H}$ is dropped in what follows to simplify the notations. The motor torque is first rewritten by eliminating the elastic torque between (1) and (2):

$$
\boldsymbol{\tau}=-\boldsymbol{\tau}_{e x t}+\mathbf{M} \ddot{\mathbf{x}}_{2}+\mathbf{I}_{m o t} \ddot{\mathbf{x}}_{1}+\mathbf{H}+\mathbf{F}_{v m} \dot{\mathbf{x}}_{1}+\mathbf{F}_{v a} \dot{\mathbf{x}}_{2}
$$

The derivatives of $x_{2}$ in (14) are then replaced by their expressions obtained from the motor equation (2) differentiated with respect to time:

$$
\begin{aligned}
& \mathbf{x}_{2}=\mathbf{x}_{1}+\mathbf{K}^{-1} \mathbf{I}_{m o t} \ddot{\mathbf{x}}_{1}-\mathbf{K}^{-1} \boldsymbol{\tau}+\mathbf{K}^{-1} \mathbf{F}_{v m} \dot{\mathbf{x}}_{1} \\
& \dot{\mathbf{x}}_{2}=\dot{\mathbf{x}}_{1}+\mathbf{K}^{-1} \mathbf{I}_{m o t} \mathbf{x}_{1}^{(3)}-\mathbf{K}^{-1} \dot{\boldsymbol{\tau}}+\mathbf{K}^{-1} \mathbf{F}_{v m} \ddot{\mathbf{x}}_{1} \\
& \ddot{\mathbf{x}}_{2}=\ddot{\mathbf{x}}_{1}+\mathbf{K}^{-1} \mathbf{I}_{m o t} \mathbf{x}_{1}^{(4)}-\mathbf{K}^{-1} \ddot{\boldsymbol{\tau}}+\mathbf{K}^{-1} \mathbf{F}_{v m} \mathbf{x}_{1}^{(3)}
\end{aligned}
$$

These expressions depend on the derivatives of the motor torque. The definition of the residual is used to relate these terms with the derivatives of the residual:

$$
\tau=\hat{\tau}-\mathbf{r} \Rightarrow \dot{\tau}=\dot{\hat{\tau}}-\dot{\mathbf{r}} \Rightarrow \ddot{\tau}=\ddot{\hat{\tau}}-\ddot{\mathbf{r}}
$$

The motor torque estimate in the residual calculations being obtained from the rigid model, the following expressions hold:

$$
\begin{aligned}
& \hat{\boldsymbol{\tau}}=\left(\hat{\mathbf{M}}+\hat{\mathbf{I}}_{m o t}\right) \ddot{\mathbf{x}}_{1}+\hat{\mathbf{H}}+\hat{\mathbf{F}}_{v} \dot{\mathbf{x}}_{1} \\
& \dot{\hat{\tau}}=\dot{\hat{\mathbf{M}}} \ddot{\mathbf{x}}_{1}+\left(\hat{\mathbf{M}}+\hat{\mathbf{I}}_{m o t}\right) \mathbf{x}_{1}^{(3)}+\dot{\hat{\mathbf{H}}}+\hat{\mathbf{F}}_{v} \ddot{\mathbf{x}}_{1} \\
& \ddot{\hat{\boldsymbol{\tau}}}=\ddot{\hat{\mathbf{M}}} \ddot{\mathbf{x}}_{1}+2 \dot{\hat{\mathbf{M}}} \mathbf{x}_{1}^{(3)}+\left(\hat{\mathbf{M}}+\hat{\mathbf{I}}_{m o t}\right) \mathbf{x}_{1}^{(4)}+\ddot{\hat{\mathbf{H}}}+\hat{\mathbf{F}}_{v} \mathbf{x}_{1}^{(3)}
\end{aligned}
$$

Finally, computing the residual as the difference between $\hat{\tau}$ and $\tau$ results in the following differential equation in $\mathbf{r}$ :

$$
\begin{aligned}
& \ddot{\mathbf{r}}+\mathbf{K M}^{-1} \mathbf{F}_{v a} \mathbf{K}^{-1} \dot{\mathbf{r}}+\mathbf{K} \mathbf{M}^{-1} \mathbf{r}=\mathbf{K} \mathbf{M}^{-1} \boldsymbol{\tau}_{e x t}+\ldots \\
& +\left(\hat{\mathbf{M}}+\Delta \mathbf{I}_{m o t}\right) \mathbf{x}_{1}^{(4)}+\left(\hat{\mathbf{F}}_{v}-\mathbf{F}_{v m}+2 \dot{\hat{\mathbf{M}}}\right) \mathbf{x}_{1}^{(3)}+\ldots \\
& +\mathbf{K M}^{-1} \mathbf{F}_{v a} \mathbf{K}^{-1}\left(\hat{\mathbf{M}}+\Delta \mathbf{I}_{m o t}\right) \mathbf{x}_{1}^{(3)}+\ldots \\
& +\mathbf{K M}^{-1}\left(\Delta \mathbf{M}_{r i g}+\mathbf{F}_{v a} \mathbf{K}^{-1}\left(\hat{\mathbf{F}}_{v}-\mathbf{F}_{v m}+\dot{\hat{\mathbf{M}}}\right)\right) \ddot{\mathbf{x}}_{1}+\ldots \\
& +\ddot{\hat{\mathbf{M}}} \ddot{\mathbf{x}}_{1}+\mathbf{K} \mathbf{M}^{-1}\left(\hat{\mathbf{F}}_{v}-\mathbf{F}_{v m}-\mathbf{F}_{v a}\right) \dot{\mathbf{x}}_{1}+\ldots \\
& +\mathbf{K M}^{-1} \boldsymbol{\Delta} \mathbf{H}+\mathbf{K M}^{-1} \mathbf{F}_{v a} \mathbf{K}^{-1} \dot{\hat{\mathbf{H}}}+\ddot{\hat{\mathbf{H}}}
\end{aligned}
$$

with $\Delta \mathbf{M}_{r i g}=\hat{\mathbf{M}}_{r i g}-\mathbf{M}_{r i g}$ and $\Delta \mathbf{I}_{m o t}=\hat{\mathbf{I}}_{m o t}-\mathbf{I}_{m o t}$. The terms $\Delta \mathbf{M}_{\text {rig }}, \Delta \mathbf{I}_{\text {mot }}, \Delta \mathbf{H}$ represent the uncertainties resulting from an imperfect knowledge of parameters as well as the use of the motor variables instead of the joint ones in the computation of the estimates.

This expression shows that in presence of unmodeled flexibilities, the residual has second order nonlinear dynamics, and is affected by the motor position and its derivatives up to the fourth order due both to the neglected flexibilities in the torque estimation based on the rigid model, as well as parametric uncertainty in the inertia matrices, $\Delta \mathbf{M}_{\text {rig }}$ and $\Delta \mathbf{I}_{m o t}$. Additional terms due to parametric uncertainty in $\mathbf{H}$ and its derivatives represent another disturbance. These observations are experimentally illustrated in the next section and are used in Section III to build a simplified model of the residual.

\section{Illustrative experimental example}

This section presents an example based on experimental data that illustrates the insufficiency of a static threshold for detection and the residual dependence on the robot's state. The experiments are performed on the ASSIST robot arm (Fig. 3), a 7-dof lightweight robot manipulator developed at CEA LIST [28], with two actuated joints $j_{1}$ (shoulder) and $j_{2}$ (elbow), the five other dof being fixed for these experiments. The ASSIST robot arm features mechanically backdrivable actuators with low friction levels, which makes it particularly suitable for human robot interaction without additional force sensors. Torque-controlled DC motors drive each joint via a cable-based actuation system. Motor shafts are equipped with position encoders, and the robot is controlled using a realtime dedicated controller running VxWorks, with a sample time of $t_{s}=4 \mathrm{~ms}$. With only joints $j_{1}$ and $j_{2}$ actuated, the robot motion is restricted to the vertical plane.

For collision detection experiments, the robot is controlled in position with a proportional-derivative (PD) control law. Two experiments are performed with the same triangular reference trajectory, which is exciting for the modeling uncertainties as it represents alternating velocity steps and acceleration impulses. Experiment 1 is collision free, while a collision of moderate amplitude is applied by the operator on the robot's second link in experiment 2, causing a small deviation in the positions when compared to the collision-free experiment (Fig. 4). This deviation is rejected by the control current (Fig.5) and is smaller than the maximum tracking error of the considered trajectory with the given PD controller. Experiments 1 and 2 being performed in the same conditions, 


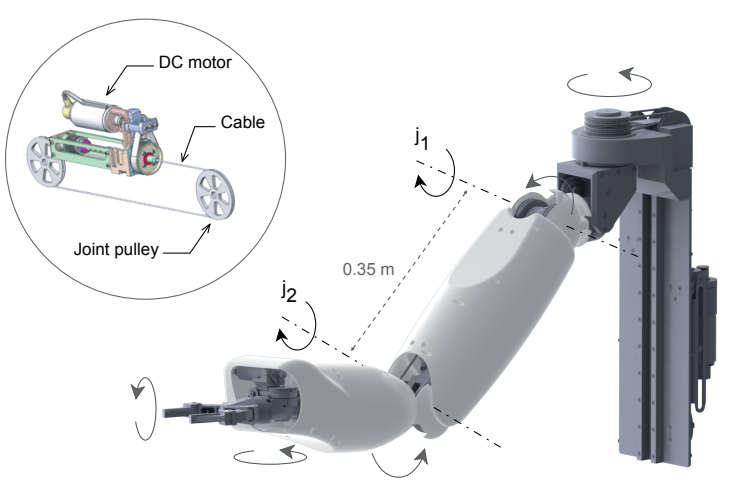

Fig. 3. 7-dof ASSIST robot arm with 2 actuated joints $j_{1}, j_{2}$ considered in this study, and cable-based actuation system.
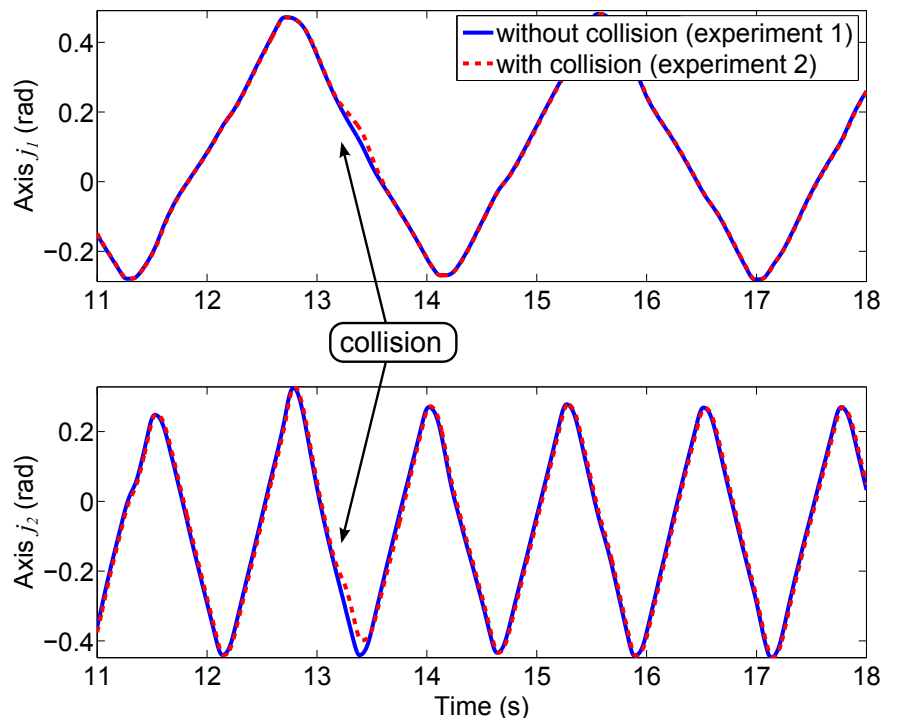

Fig. 4. Motor position $\mathbf{x}_{1}$ in experiment 1 (collision-free, solid line) and experiment 2 (collision of moderate amplitude applied to the robot, dashed line).

the motor current references are coincident within the robot's repeatability range, except for the collision.

The corresponding residual is computed according to the definition (7) and represented Fig. 6. The deviation produced by the collision is too small to be detected when compared with a static threshold fixed above the current extrema or the maximum residual amplitudes obtained on this trajectory. Observed residual peaks reflect a dependence of the residual on the robot's state, which is illustrated in Fig. 7 and Fig. 8 respectively for acceleration and speed using a third trajectory tracking experiment, with a triangular position reference modulated in frequency (equivalent to alternating velocity steps of varying amplitude). The observed dependence is due to parametric uncertainties on the inertia matrix and friction, as well as joint flexibilities which are not taken into account in the residual computations.

As an alternative solution to further model refinement and identification, the dependence of the residual on the robot state due to uncertainties in the model will be treated by filtering and a suitably designed dynamic threshold, based on a local linear
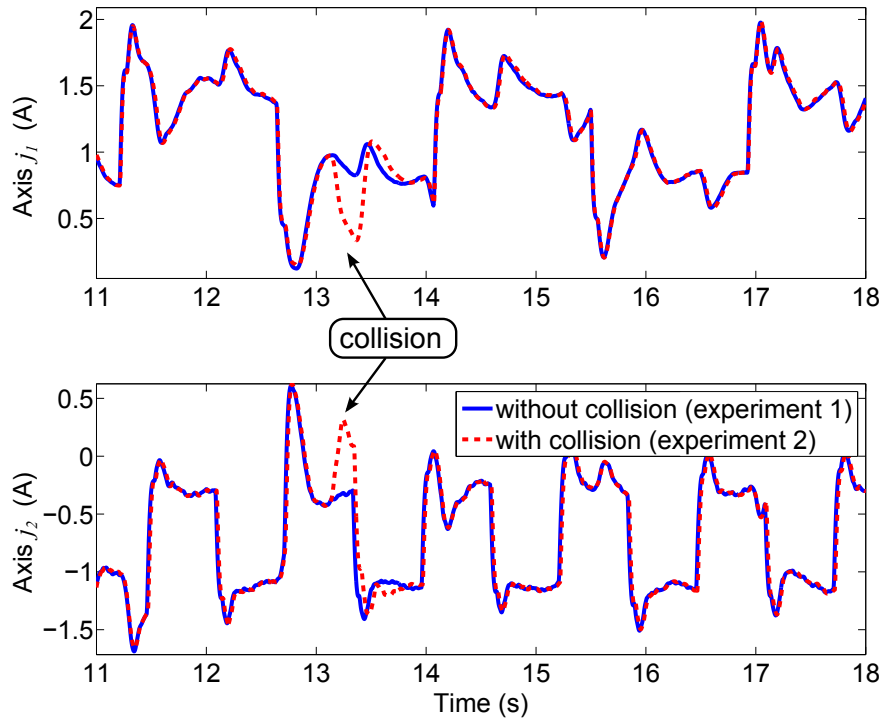

Fig. 5. Motor control current $\mathbf{i}_{m}$ in experiment 1 (collision-free, solid line) and experiment 2 (collision of moderate amplitude applied to the robot, dashed line).
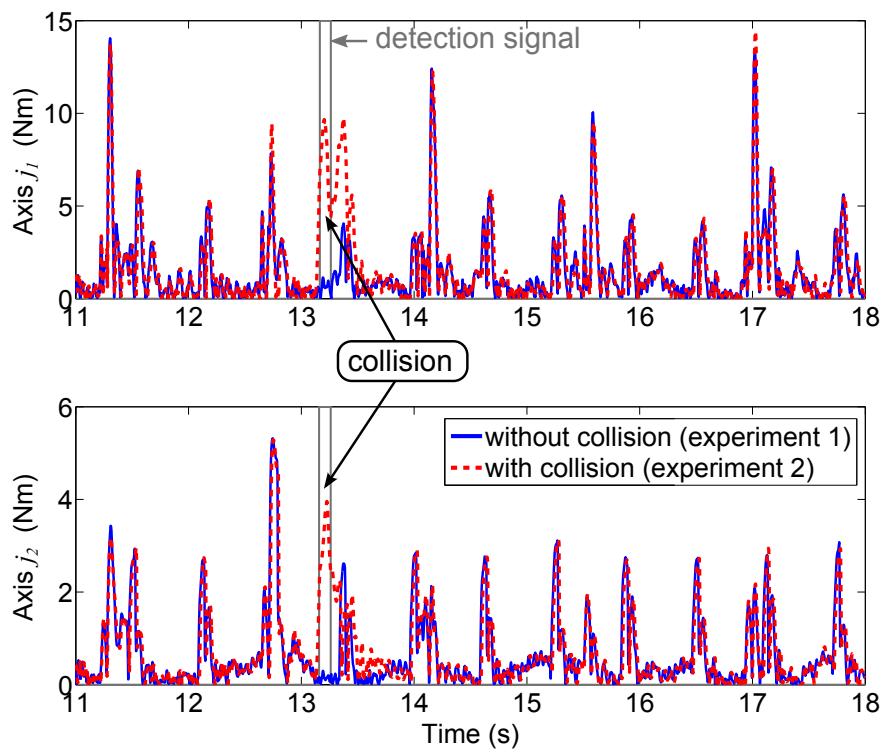

Fig. 6. Residual $\mathbf{r}$ in experiment 1 (collision-free, solid line) and experiment 2 (collision of moderate amplitude applied to the robot, dashed line). The detection signal produced by the proposed method (see Section IV-A) is reported for information only.

model of the residual dynamics (22). Adaptive estimation of filter coefficients is used to take into account the time varying nature of this approximate model due to nonlinearities and changing operating conditions.

\section{PRoposed RESIDUAL EVALUATION METHOD}

This section details the proposed method of residual evaluation based on filtering and dynamic threshold, and its adaptive formulation.

\section{A. Modeling of the residual}

The physical models (10) and (22) obtained under the rigid and flexible-joint assumptions of the robot dynamics in 


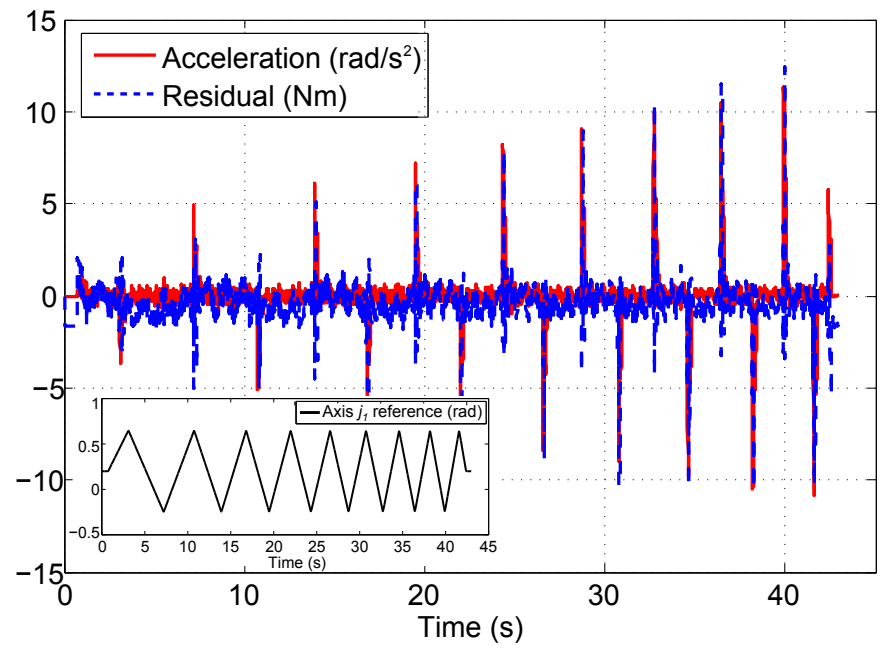

Fig. 7. Illustration of the dependence of the residual $\mathbf{r}$ on acceleration for axis $j_{1}$ with corresponding position reference signal (experiment 3 ).

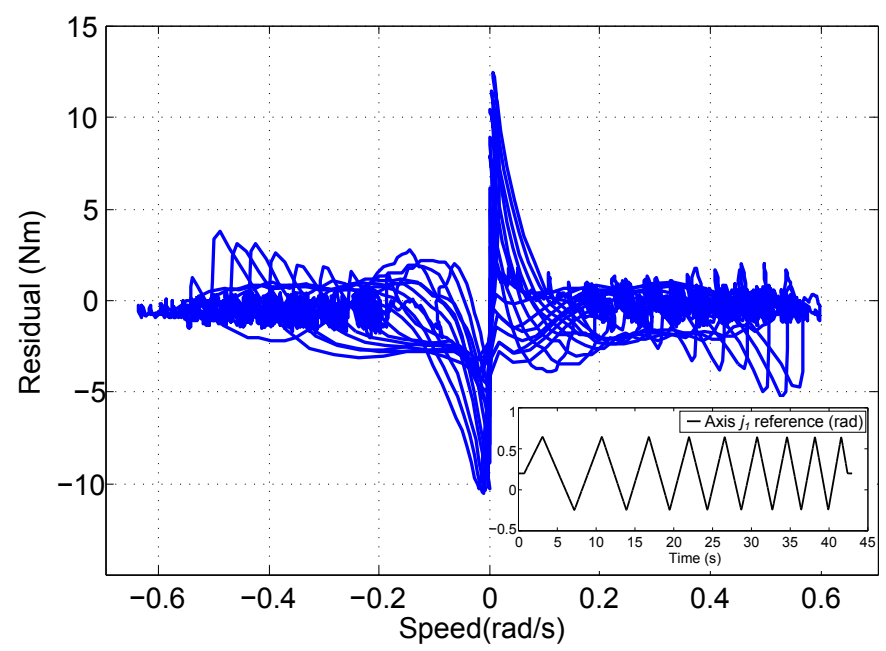

Fig. 8. Illustration of the dependence of the residual $\mathbf{r}$ on speed for axis $j_{1}$ with corresponding position reference signal (experiment 3 ).

presence of uncertainties are both used to gain insight in the residual behavior, and to select the overall structure and the inputs of a simplified model. However, the inner structure of each input influence is not physically modeled but considered as black-box transfer functions, which orders are seen as design parameters, and which coefficients are estimated online.

Locally around a fixed configuration, the model (22) can be considered as linear with respect to the residual and the motor position, and rewritten in discrete time at instant $k$ ( $z$ denoting the discrete variable):

$$
\mathbf{G}_{0}(z) \mathbf{r}(k)=\boldsymbol{\tau}_{\text {ext }}(k)+\mathbf{f}\left(\mathbf{x}_{1}^{(4)}, \mathbf{x}_{1}^{(3)}, \ddot{\mathbf{x}}_{1}, \dot{\mathbf{x}}_{1}\right)+\mathbf{d}(k)
$$

where $\mathbf{G}_{0}(z) \in \mathbb{R}^{n \times n}$ denotes a transfer matrix expressing the influence of the flexible transmission dynamics, $f$ a vector function regrouping the contributions due to the motor position $\mathbf{x}_{1}$ and its derivatives, and $\mathbf{d}$ a disturbance signal regrouping the influence of other terms.

From the previous equation, the following simplified repre- sentation of the residual dynamics is chosen as the basis of proposed approach:

$$
\mathbf{G}(z) \mathbf{r}(k)=\boldsymbol{\tau}_{\text {ext }}(k)+\sum_{i=1}^{m} \mathbf{T}_{i}(z) \mathbf{u}_{i}(k)+\mathbf{b}(k)
$$

We define the left hand side of (24) as $\tilde{\mathbf{r}}=\mathbf{G}(z) \mathbf{r}(k)$, with $\mathbf{G} \in \mathbb{R}^{n \times n}$ a transfer matrix. The filtered residual $\tilde{\mathbf{r}}$ is thus considered to be dependent of $m$ different input types $\mathbf{u}_{i} \in \mathbb{R}^{n}$, which are functions (possibly nonlinear, see Section II-B) of different robot's states. For instance, to illustrate the dependence of the residual on acceleration and sign of speed (see Fig. 7 and 8), one can consider $\mathbf{u}_{1}=\ddot{\mathbf{x}}_{1}$ and $\mathbf{u}_{2}=\operatorname{sign}\left(\dot{\mathbf{x}}_{1}\right)$ with $m=2$. The way in which inputs $\mathbf{u}_{i}$ affect $\tilde{\mathbf{r}}$ is modeled by transfer matrices $\mathbf{T}_{i}(z) \in \mathbb{R}^{n \times n}$. Additional term $\mathbf{b} \in \mathbb{R}^{n}$ is considered to represent white noise contributions vector, possibly of different variances over the different robot's axes. Note that transfer matrices in (24) are time varying to take into account model nonlinearities.

From the right hand side of (24), a dynamic threshold $\mathbf{v}_{d y n}(k)$ is defined by:

$$
\mathbf{v}_{d y n}(k)=\sum_{i=1}^{m} \mathbf{T}_{i}(z) \mathbf{u}_{i}(k)
$$

In the collision free case, equation (24) thus becomes:

$$
\tilde{\mathbf{r}}(k)=\mathbf{G}(z) \mathbf{r}(k)=\mathbf{v}_{d y n}(k)+\mathbf{b}(k)
$$

In the proposed approach, coefficients of $\mathbf{G}(z)$ and $\mathbf{T}_{i}(z)$ are recursively estimated in real-time and used to compute $\tilde{\mathbf{r}}$ and $\mathbf{v}_{\text {dyn }}$. These signals are used to detect the collision as detailed in the following section.

\section{B. Detection method}

To isolate the effects of $\tau_{\text {ext }}$, the filtered residual $\tilde{\mathbf{r}}$ is compared with a dynamic threshold built from $\mathbf{v}_{d y n}$ and a static term $\mathbf{v}_{\text {stat }}$ greater than the variance of $\mathbf{b}$ (Fig. 9) :

$$
\mathbf{v}(k)=\mathbf{v}_{d y n}(k)+\mathbf{v}_{\text {stat }}
$$

The comparison method is detailed in Section III-D. Note that the proposed evaluation approach focuses on dynamic impact detection. Indeed, the calculation of $\tilde{\mathbf{r}}$ consists in filtering the residual by $\mathbf{G}(z)$. From the residual dynamics previously highlighted in the flexible-joint case, $\mathbf{G}(z)$ is expected to have high-pass characteristics. It has the essential advantage of eliminating the influence of static uncertainties like offsets due to gravity errors when applied to $\mathbf{r}$.

The high-pass nature of $\mathbf{G}(z)$ applied to $\mathbf{r}$ has consequences on the characteristics of collision signals that can be detected. The proposed method ensures a fine detection of dynamic impacts (see [6] for a classification of impacts and associated injury risks), while detection of slow collisions assimilated to static loading, which can be of high amplitude and dangerous in case of clamping, requires an additional evaluation method. The latter can possibly consist of a simple comparison with a static threshold without filtering as shown in Fig. 10. 


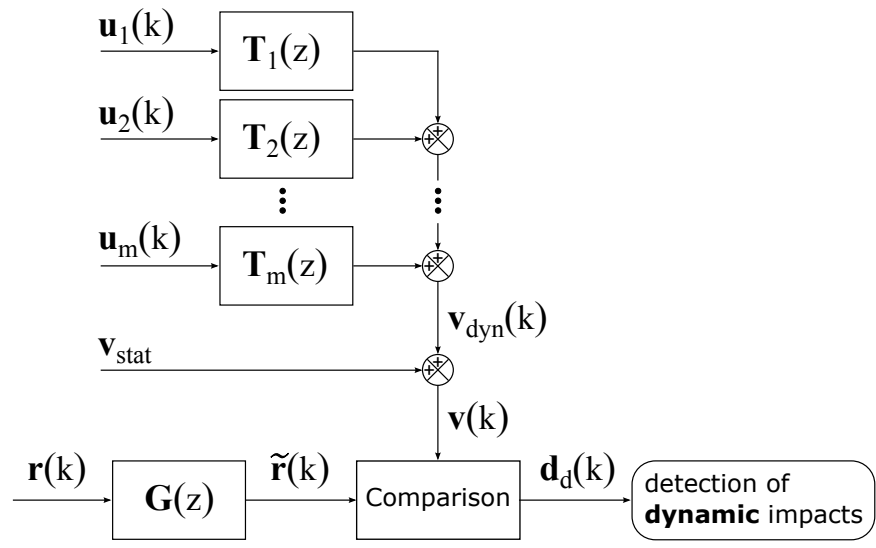

Fig. 9. Residual evaluation method for detection of dynamic impacts: residua filtering and comparison with a state dependent dynamic threshold.

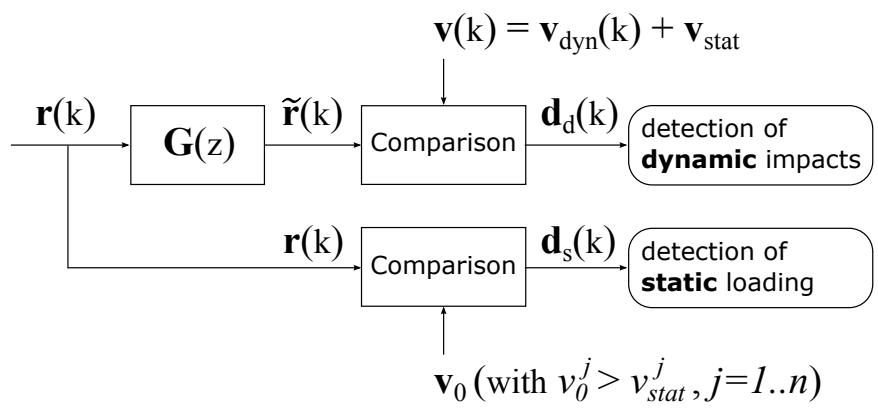

Fig. 10. Detection of dynamic impacts (proposed method) and static loading.

\section{Adaptive formulation}

The coefficients of filter $\mathbf{G}(z)$ and transfer matrices $\mathbf{T}_{i}(z)$ are not known in practice. In the following, an adaptive procedure is proposed for their on-line estimation. The detection algorithm being applied axis by axis, the computations below are detailed for the $j$-th axis.

1) Difference equation: In order to simplify real-time computations, $\mathbf{r}$ is considered as an autoregressive process, and $\mathbf{G}(z) \in \mathbb{R}^{n \times n}$ is assumed to be a diagonal matrix of polynomials in $z$ :

$$
\begin{aligned}
\mathbf{G}(z) & =\left[\begin{array}{ccc}
G_{1}(z) & \ldots & 0 \\
& \ddots & \\
0 & \ldots & G_{n}(z)
\end{array}\right] \\
G_{j}(z) & =1+\gamma_{1}^{j} z^{-1}+\ldots+\gamma_{\eta}^{j} z^{-\eta}, j=1 . . n
\end{aligned}
$$

For the $j$-th joint, equation (26) can be rewritten as a difference equation, with $r_{j}(k)$ depending on the past values of $r_{j}$ :

$$
\begin{aligned}
& G_{j}(z) r_{j}(k)=v_{d y n}^{j}(k)+b_{j}(k) \\
& \Rightarrow r_{j}(k)=\left(\phi_{G}^{j}(k)\right)^{T} \boldsymbol{\theta}_{G}^{j}+v_{d y n}^{j}(k)+b_{j}(k)
\end{aligned}
$$

with $\phi_{G}^{j}(k)=\left[r_{j}(k-1) \ldots r_{j}(k-\eta)\right]^{T}$ and the parameter vector $\boldsymbol{\theta}_{G}^{j}=\left[-\gamma_{1}^{j} \ldots-\gamma_{\eta}^{j}\right]^{T}$. Equation (31) can be further expanded by expressing $v_{d y n}^{j}(k)$ as a function of past values of inputs $\mathbf{u}_{i}$. Let the $j$-th line of the transfer matrix $\mathbf{T}_{i}$ be denoted $\mathbf{T}_{i}^{j}(z)$, and its coefficients regrouped in the parameter vector $\boldsymbol{\theta}_{v}^{j}$. The difference equation for $v_{d y n}^{j}(k)$ is obtained from its definition with respect to its past values, as well as the present and past values of inputs $\mathbf{u}_{i}$ :

$$
\begin{aligned}
& v_{d y n}^{j}(k)=\sum_{i=1}^{m} \mathbf{T}_{i}^{j}(z) \mathbf{u}_{i}(k) \\
& \Rightarrow v_{d y n}^{j}(k)=\left(\phi_{v}^{j}(k)\right)^{T} \boldsymbol{\theta}_{v}^{j}
\end{aligned}
$$

A detailed expression of $\phi_{v}^{j}(k)$ is provided in Appendix A. Expressing the regression (33) with respect to past values of the output $v_{d y n}^{j}$, which are computed using previously estimated coefficients values, introduces additional degrees of freedom in the algorithm. This prevents the collision from being identified as model variations, and ensures that the adaptation and the detection can be performed at the same time.

The global difference equation for $r_{j}(k)$ can therefore be written in the linear regression form as follows, the parameter vector $\boldsymbol{\theta}_{r}^{j}$ to be estimated regrouping both $\boldsymbol{\theta}_{G}^{j}$ and $\boldsymbol{\theta}_{v}^{j}$ :

$$
\begin{aligned}
& r_{j}(k)=\left(\phi_{G}^{j}(k)\right)^{T} \boldsymbol{\theta}_{G}^{j}+\left(\phi_{v}^{j}(k)\right)^{T} \boldsymbol{\theta}_{v}^{j}+b_{j}(k) \\
& r_{j}(k)=\left(\boldsymbol{\phi}_{r}^{j}(k)\right)^{T} \boldsymbol{\theta}_{r}^{j}+b_{j}(k)
\end{aligned}
$$

These notations are made explicit for a 2-dof robot example in Appendix B.

2) Recursive estimation: Recursive Least Squares (RLS) with constant forgetting factor are applied to recursively estimate $\boldsymbol{\theta}_{r}^{j}$ for each axis $j$ according to (35). The minimized cost function $J_{j}$ at instant $k$ is:

$$
\begin{aligned}
& \min _{\hat{\theta}(k)} J_{j}(k) \\
& J_{j}(k)=\sum_{i=1}^{k} \lambda^{k-i}\left[y(i)-\boldsymbol{\phi}^{T}(i-1) \hat{\boldsymbol{\theta}}(k)\right]^{2}
\end{aligned}
$$

where $y=r_{j}, \hat{\boldsymbol{\theta}}=\hat{\boldsymbol{\theta}}_{r}^{j}, \boldsymbol{\phi}=\phi_{r}^{j}$. The parameter vector estimate $\hat{\boldsymbol{\theta}}(k)$ at instant $k$ is obtained recursively according to the following standard RLS equations:

$$
\begin{aligned}
\hat{\boldsymbol{\theta}}(k) & =\hat{\boldsymbol{\theta}}(k-1)+\mathbf{P}(k) \boldsymbol{\phi}(k) \epsilon^{0}(k) \\
\mathbf{P}(k) & =\frac{1}{\lambda}\left(\mathbf{P}(k-1)-\frac{\mathbf{P}(k-1) \boldsymbol{\phi}(k) \boldsymbol{\phi}^{T}(k) \mathbf{P}(k-1)}{\lambda+\boldsymbol{\phi}^{T}(k) \mathbf{P}(k-1) \boldsymbol{\phi}(k)}\right) \\
\epsilon^{0}(k) & =y(k)-\hat{\boldsymbol{\theta}}^{T}(k-1) \boldsymbol{\phi}(k)
\end{aligned}
$$

Obtained coefficients are used to compute $\tilde{\mathbf{r}}(k)=\hat{\mathbf{G}}(z) \mathbf{r}(k)$ and $\mathbf{v}_{d y n}(k)$, which are then compared.

\section{Comparison and decision block}

The comparison stage is crucial in the elaboration of the detection signal in the proposed method. In order to robustify the detection against false alarms, rather than a direct comparison of absolute values of $\tilde{\mathbf{r}}(k)$ and $\mathbf{v}(k)=\mathbf{v}_{\text {dyn }}(k)+\mathbf{v}_{\text {stat }}$, an evaluation method based on low-pass filtering of $\tilde{\mathbf{r}}$ and $\mathbf{v}_{\text {dyn }}$ and the root mean square (RMS) value is employed (Fig. 11). Low pass filters attenuate high frequency noise on $\tilde{\mathbf{r}}$, which results in a band-pass filtering of $\mathbf{r}$ and thus isolates the dynamic effects of collisions. RMS averaging of signals over time introduces in the considered detection context a dependence of the decision on a finite time horizon, which 


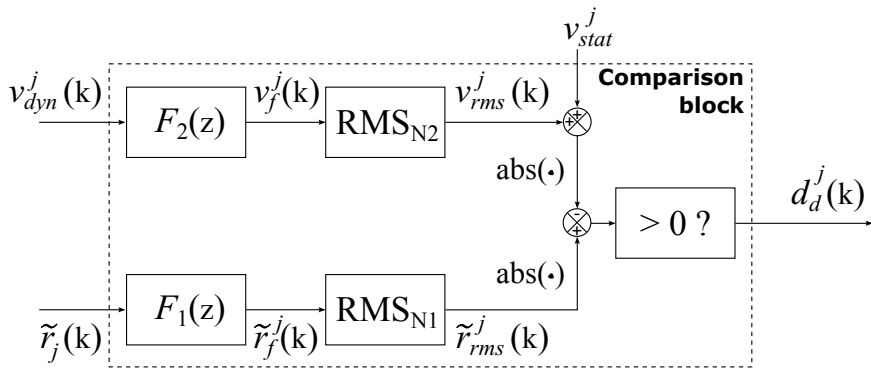

Fig. 11. Comparison of $\tilde{r}_{j}(k)$ and $v_{d y n}^{j}(k)$ for joint $j$, and resulting decision signal $d_{d}^{j}(k)$.

confers more robustness and prevents remaining false alarms. The static threshold $\mathbf{v}_{\text {stat }}$ is used in the last step of comparison of the thus obtained signals, and is chosen in accordance to the variance of the residual in absence of peaks due to acceleration, speed and collision. The evaluation is performed in an axis-by-axis manner, and the following details hold for the $j$-th axis.

In Fig. 11, $\tilde{\mathbf{r}}$ and $\mathbf{v}_{d y n}$ are filtered by first order low-pass filters of unitary gain, respectively $F_{1}(z)$ of cut-off frequency $\omega_{1}$, and $F_{2}(z)$ of cut-off frequency $\omega_{2}$. The cut-off frequencies must be selected such that $\omega_{2}>\omega_{1}$ to avoid any delay of the threshold over the residual. The higher are the cut-off frequencies, the faster the detection algorithm reacts, but the higher is the false alarm rate. If these frequencies are too low, detectability may be lost since $\tilde{\mathbf{r}}$ contains high-pass filtered residuals.

The RMS value over a time horizon is computed for the previously filtered signals (Fig. 11). The time horizon is denoted $N_{1}$ for $\tilde{\mathbf{r}}_{f}$ and $N_{2}$ for $\mathbf{v}_{f}$. For the residual, $\tilde{r}_{r m s}^{j}(k)$ is computed according to:

$$
\tilde{r}_{r m s}^{j}(k)=\sqrt{\frac{1}{N_{1}} \sum_{i=1}^{N_{1}}\left(\tilde{r}_{f}^{j}(k-i)\right)^{2}},
$$

and $v_{r m s}^{j}$ is obtained similarly from $v_{f}^{j}$. The values of $N_{1}$ and $N_{2}$ are selected such that $N_{2} \geq N_{1}$ to produce a smoother threshold that describes the filtered residual without false alarms in the absence of collision.

Finally, the logical detection signal that indicates the presence of a collision is computed for the $j$-th axis after a comparison of the previously obtained signals $\tilde{\mathbf{r}}_{r m s}$ and $\mathbf{v}_{r m s}$ at instant $k$ :

$$
d_{d}^{j}(k)= \begin{cases}1, & \text { if }\left|\tilde{r}_{r m s}^{j}(k)\right|>\left|v_{s t a t}^{j}+v_{r m s}^{j}(k)\right| \\ 0, & \text { otherwise. }\end{cases}
$$

\section{EXPERIMENTS}

In this section, the proposed algorithm is experimentally evaluated on the lightweight and backdrivable ASSIST robot arm with two actuated joints $j_{1}$ and $j_{2}$ (Fig. 3). First, results are presented for experiment 2 introduced in Section II-D. Then, the sensitivity of the detection is analyzed using an external force sensor to quantify the contact force when a detected collision prompts a transition from position controlled mode to a gravity compensated transparent mode.

The chosen inputs and filter orders in the implemented algorithm correspond to the 2-dof example described in Appendix B. The design parameters are summarized in Table I. Note that to avoid noise, inputs $\mathbf{u}_{i}$ are defined with respect to the reference trajectory. This also has the advantage of introducing anticipation in the threshold, which helps avoiding false alarms. The sign function used to compute $\mathbf{u}_{2}$ is approximated by the hyperbolic tangent function in the realtime computations to preserve signal continuity.

TABLE I

\begin{tabular}{|c|c|c|}
\hline Parameter & Notation & Parameter values \\
\hline Degree of $G_{j}(z)$ & $\eta$ & $\eta=2$ \\
\hline Dynamic threshold inputs & $u_{i, i=1 . . m}$ & $u_{1}=\operatorname{sign}\left(\dot{\mathbf{x}}_{1}^{\text {ref }}\right), u_{2}=\ddot{\mathbf{x}}_{1}^{\text {ref }}$ \\
\hline $\begin{array}{l}\text { Degree of } B_{i}(z) \text { (dynamic } \\
\text { threshold) }\end{array}$ & $\rho_{i, i=1 . . m}$ & $\rho_{1}=2, \rho_{2}=4$ \\
\hline $\begin{array}{l}\text { Degree of } A_{j}(z) \text { (dynamic } \\
\text { threshold) }\end{array}$ & $p$ & $p=20$ \\
\hline Cut-off freq. of $F_{1,2}(z)$ & $\omega_{1,2}$ & $\omega_{1}=10 \mathrm{~Hz}, \omega_{2}=50 \mathrm{~Hz}$ \\
\hline RMS horizon & $N_{1,2}$ & $N_{1}=10, N_{2}=40$ \\
\hline Static thresholds & $v_{\text {stat }}$ & $\mathbf{v}_{\text {stat }}=[0.45 ; 0.2]$ \\
\hline RLS initialization & \multicolumn{2}{|c|}{$\lambda=0.999, \mathbf{P}(1)=10 \mathbb{I}, \hat{\boldsymbol{\theta}}(1)=\mathbf{0}$} \\
\hline
\end{tabular}

DESIGN PARAMETERS OF IMPLEMENTED ALGORITHM IN THE 2-DOF CASE.

\section{A. Illustrative experimental example (continued)}

The signals involved in detection with the proposed method and corresponding to experiment 2 (Section II-D) are represented in Fig. 12. The collision occurs at 13.133s (deviation of the residual from its collision-free value in experiment 1).
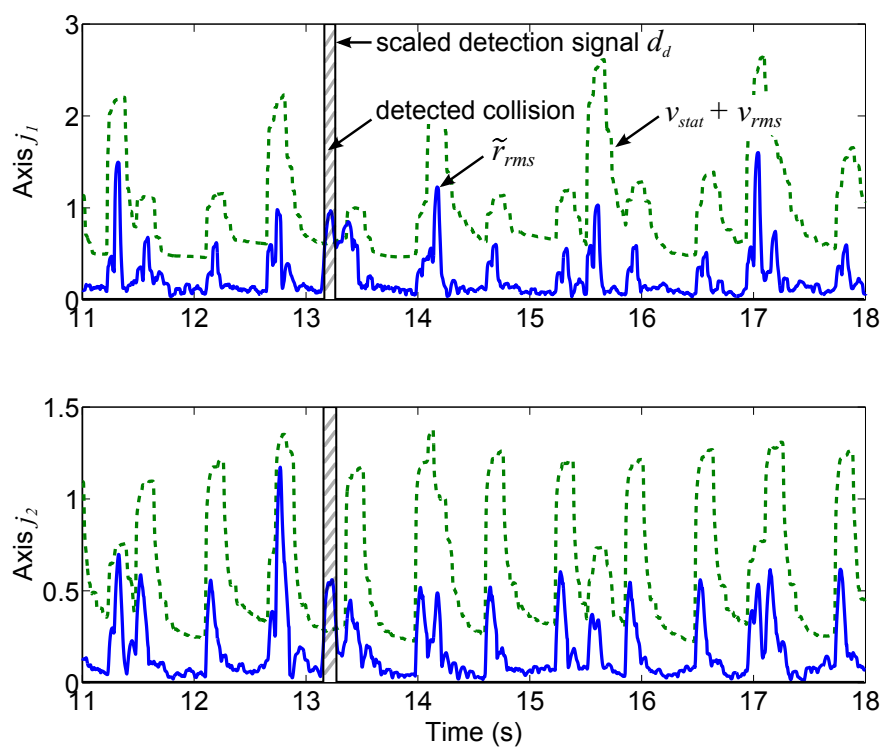

Fig. 12. Comparison of $\tilde{\mathbf{r}}_{r m s}$ (solid line) and $\mathbf{v}_{\text {stat }}+\mathbf{v}_{r m s}$ (dashed line) in experiment 2 (Section II-D), and resulting detection signal. 

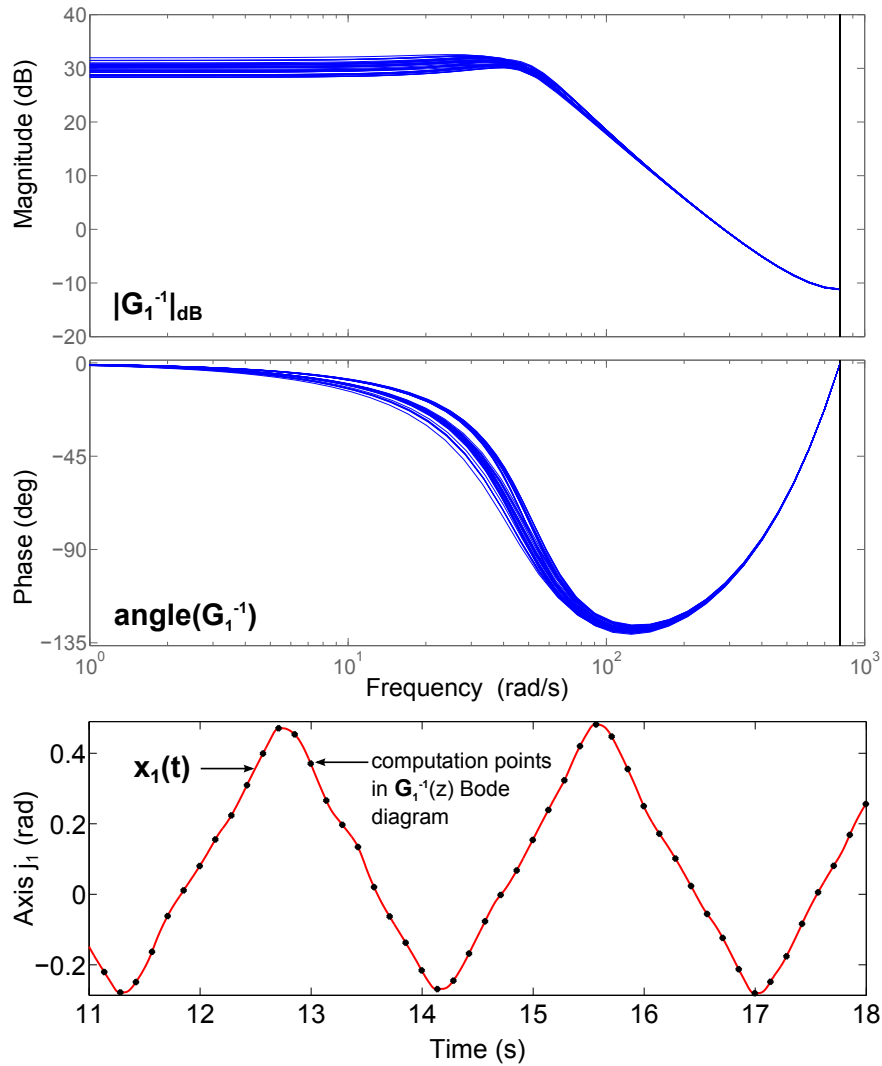

Fig. 13. Bode diagram of $G_{1}^{-1}(z)$ in experiment 2 (Section II-D) estimated on-line at different time steps along the represented motor trajectory.

The collision is first detected at 13.160 s on axis $j_{2}$, then at 13.168 s on axis $j_{1}$ (two sample periods later). The minimum detection time on this specific example is therefore $0.027 \mathrm{~s}$. A secondary detection occurs on axis $j_{1}$ right after the first but is not represented for clarity.

Variations with time of the on-line estimated filter $G_{1}(z)$ are illustrated in Fig. 13, at 50 different time instants along the reference triangular trajectory. It can be noticed that the main variations affect the filter static gain, while the cut-off frequency remains close to $50 \mathrm{rad} / \mathrm{s}$.

\section{B. Evaluation with force sensor}

In this section, the sensitivity of the proposed detection method, i.e. the minimal detected force, is experimentally evaluated for collisions with environments of different stiffnesses [28]. The axis $j_{1}$ of the ASSIST robot arm is actuated along a triangular trajectory around the horizontal configuration with fully extended arm $\left(\mathbf{x}_{2}=[0,0]^{T}\right)$. The test is performed for different trajectories amplitudes, allowing to control the speed at the moment of the collision.

During the downward movement of the robot arm, a collision occurs between the end-effector of the robot and an elastomer of known stiffness. Several elastomers of different stiffnesses are tested. A load-cell (FUTEK LSB200 10lb) is placed under the elastomer to measure the collision force (Fig. 14). This sensor is not used in the detection algorithm, but only for validation purposes. The collision detection strategy is thus tested for different speeds and stiffnesses, allowing

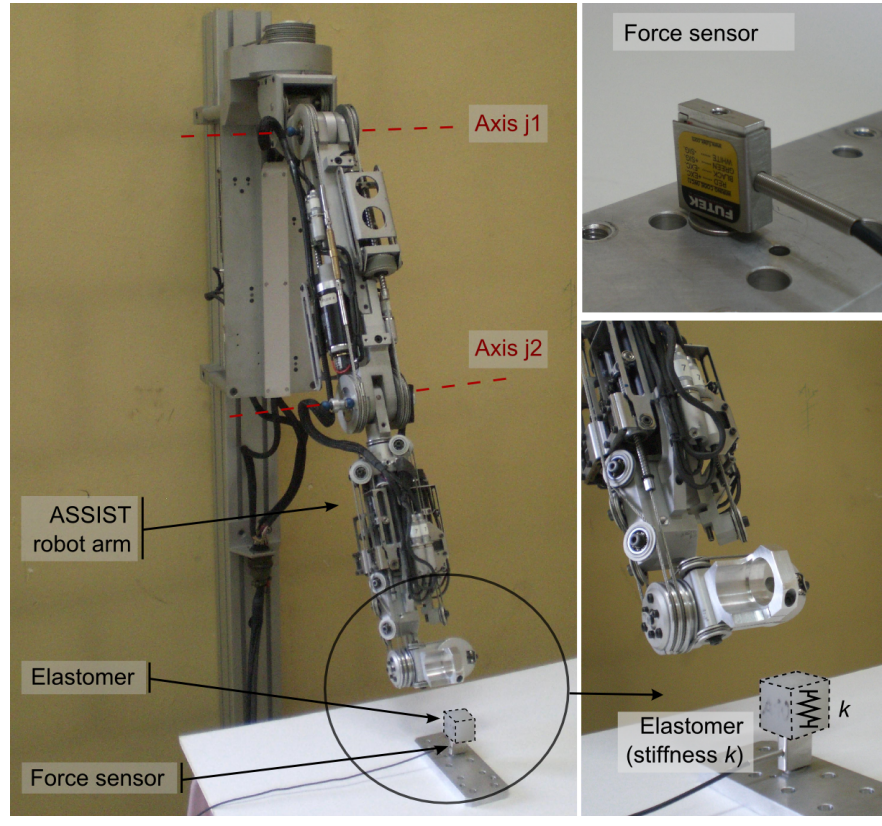

Fig. 14. ASSIST robot arm and experimental setup with force sensor used for sensitivity evaluation.

to evaluate the algorithm in different operating conditions.

An example of a collision detected on axis $j_{2}$ is represented in Fig. 15, which summarizes different signals involved in the detection algorithm for both axes (the filtered residual to be compared with the dynamic threshold, and the resulting detection logical signal) and the measured contact force. The dynamic threshold displays a first peak (zone I) caused by the initial convergence of the adaptive algorithm. Zone II corresponds to a velocity sign reversal and displays a peak in the residual due to uncertainties, which is also described by the dynamic threshold. Once the collision is detected (zone III), the robot arm is set in a gravity compensation mode and the measured collision force decreases.

Table II illustrates the improvement in the sensitivity of the algorithm when compared with a direct comparison of the residual with a static threshold. Such a threshold would allow detection only of forces greater than $20 \mathrm{~N}$ to avoid false alarms due to acceleration peaks occurring on the test trajectory, whereas the proposed algorithm allows to detect forces as small as $5 \mathrm{~N}$. Table II also brings out a dependency of the new algorithm on the stiffness. Higher elastomer stiffnesses lead to smaller minimal detected forces. Due to high pass filtering of the residual, the algorithm is therefore particularly efficient with relatively rigid contact. Note that the maximal tested stiffness $k_{1}=10.12 \mathrm{~N} / \mathrm{mm}$ corresponds to the minimal stiffness of the human body (neck, belly) according to [7].

\section{COMMENTS AND CONCLUSION}

In this article, a new collision detection method was presented for flexible-joint robots, based on a residual evaluation method explicitly dealing with modeling uncertainties. The proposed method uses only motor-side information, and thus requires no additional sensors. No refined model of flexibilities or friction is necessary since the residual generation is based 
TABLE II

MINIMAL DETECTED FORCES WITH PROPOSED METHOD FOR DIFFERENT ELASTOMER STIFFNESSES, AND WITH A SIMPLE STATIC THRESHOLD $\mathbf{v}_{0}$.

\begin{tabular}{llll}
\hline & $\begin{array}{l}\text { Environment } \\
\text { stiffness }(\mathrm{N} / \mathrm{mm})\end{array}$ & $\begin{array}{l}\text { Minimal detected } \\
\text { force }(\mathrm{N})\end{array}$ & $\begin{array}{l}\text { Equivalent } \\
\text { mass }(\mathrm{kg})\end{array}$ \\
\hline Proposed method & $k_{1}=10.12$ & 4.44 & 0.452 \\
& $k_{2}=5.93$ & 5.61 & 0.572 \\
& $k_{3}=2.12$ & 5.87 & 0.598 \\
\hline Static threshold $\mathbf{v}_{0}$ & & $>20$ & $>1.962$ \\
\hline
\end{tabular}

on the standard rigid robot dynamic model. The theoretic expression of residual dynamics resulting from transmission flexibilities and parametric uncertainties was obtained and used to define a simplified linear modeling of the residual. On the basis of this model, the proposed evaluation algorithm consists in filtering and comparison with a state dependent dynamic threshold. On-line estimation of filter coefficients ensures the adaptation of the algorithm to different operating conditions and accounts for nonlinearities.

Experimental results show a significantly better detection sensitivity of dynamic impacts when compared to a static threshold. Collision forces as small as $5 \mathrm{~N}$ can be detected while maintaining the robustness to parametric uncertainty, and the adaptive algorithm avoids extensive experiments for the parametric identification of the model. While a set of representative motions over the whole robot's workspace is still needed for a better adjustment of high level design parameters like static threshold $\mathbf{v}_{\text {stat }}$ (see Table I), the thus tuned parameter values are not restricted to a single trajectory type. For the adjustment of these high level parameters, optimization-based selection of representative trajectories can be envisaged for a reduced number of experiments.
The adaptive character of the proposed algorithm is also beneficial for operation with unmodeled payloads. Indeed, an unknown constant payload fixed at the end of the robot can be seen as an uncertainty on the robot's dynamic parameters due to a change in the mass properties of the last link. The detection algorithm being designed to be robust to model uncertainties, load-induced bounded model variations are filtered by the algorithm without any specific algorithm modification.

Detection sensitivity is influenced by several factors. A first factor is the robot configuration, since external forces are only detected from their repercussion on the motor torques through the Jacobian matrix. Its effects do not depend on the residual evaluation method, but only on the robot structure, with forces belonging to the Jacobian matrix kernel structurally impossible to detect. In favorable configurations however, backdrivable robots are of major interest since external forces are reflected with high fidelity on the motor torques, providing the ability to detect collisions that occur not only at the end-effector but on the whole robot's body. Another detection sensitivity factor, proper to the proposed algorithm, is related to the dynamic characteristics of the impact. Detection performances depend of the environment stiffness due to residual band-pass filtering, thus leading to different levels of minimal detected forces. Future research directions include investigations on the relation between detection sensitivity, environment stiffness and detailed impact properties like speed and energy, as well as further experimental evaluations of the algorithm under varying payload.

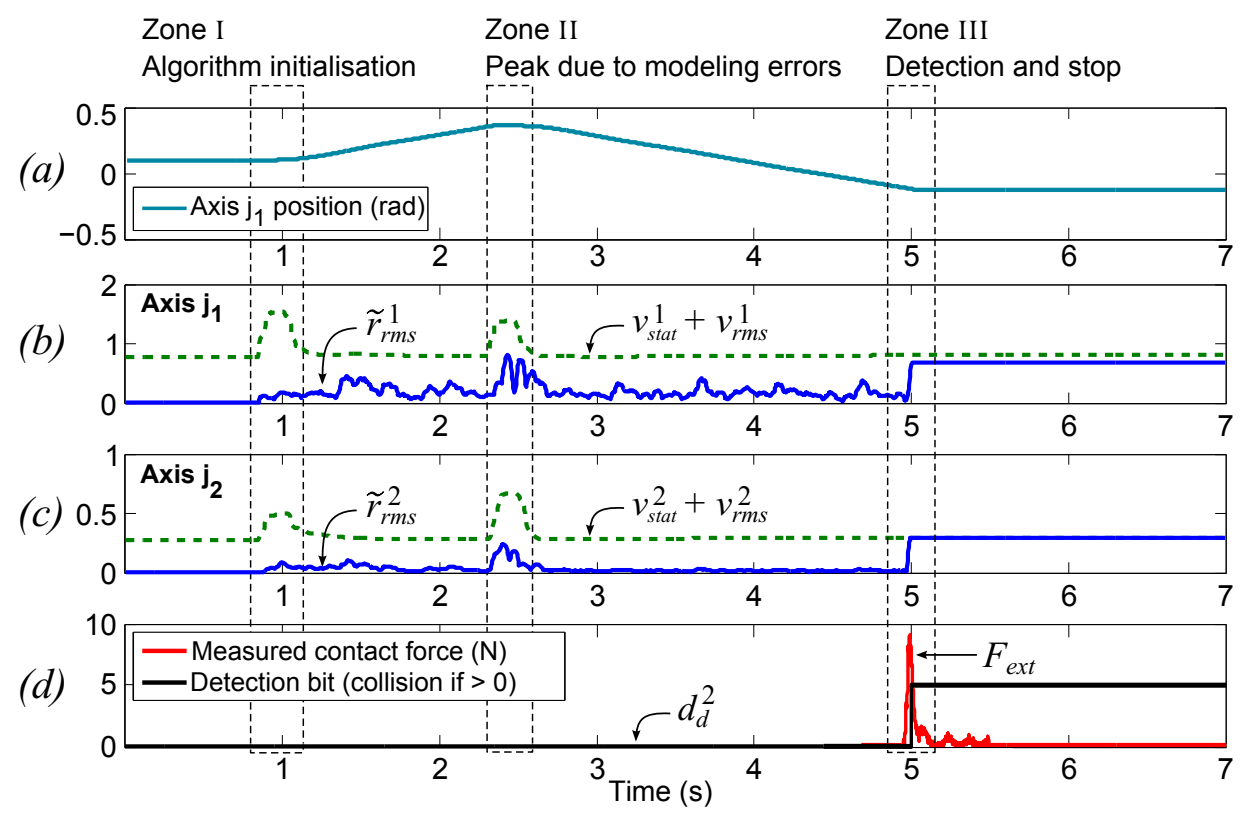

Fig. 15. Collision detection experiment with transition from position controlled mode to safe gravity compensated mode; collision is detected on axis $j_{2}$. (a) Reference trajectory for $j_{1}$ position; $(b)$ and $(c)$ RMS values of the filtered residual and dynamic threshold for $j_{1}$ and $j_{2}$; $(d)$ Measured contact force and detection signal. 


\section{APPENDIX A}

Expression of $\varphi_{\mathbf{v}}^{\mathbf{j}}(\mathbf{k})$ : For a $n$-dof robot, the vector $\mathbf{v}_{d y n}$ is decomposed in its components for each axis :

$$
\mathbf{v}_{d y n}(k)=\left[\begin{array}{lllll}
v_{d y n}^{1} & \ldots & v_{d y n}^{j} & \ldots & v_{d y n}^{n}
\end{array}\right]^{T} \in \mathbb{R}^{n}
$$

For each axis, the dynamic threshold is computed as the output of a MISO filter, which inputs are the $m$ vectors $\mathbf{u}_{i} \in \mathbb{R}^{n}$ corresponding each to a specific type of robot's state (e.g. acceleration). Note that for a given input type $\mathbf{u}_{i}$, measures from all $n$ axes contribute to the computation of the $j$-th component of $\mathbf{v}_{d y n}$. The $j$-th component of $\mathbf{v}_{d y n}$ corresponding to the $j$-th axis is modeled as follows :

$$
\begin{aligned}
v_{d y n}^{j}(k) & =\frac{1}{A_{j}(z)} \sum_{i=1}^{m} \mathbf{B}_{i}^{j}(z) \mathbf{u}_{i}(k) \\
& =\frac{1}{A_{j}(z)} \sum_{i=1}^{m} \sum_{l=1}^{n} B_{i_{l}}^{j}(z) u_{i_{l}}(k)
\end{aligned}
$$

with the same denominator $A_{j}(z), \mathbf{B}_{i}^{j}(z) \in \mathbb{R}^{1 \times n}$ a matrix of polynomial components $B_{i_{l}}^{j}(z)$, and the input vector $\mathbf{u}_{i}(k) \in$ $\mathbb{R}^{n}$ of scalar components $u_{i_{l}}(k)$.

Regrouping the coefficients of $A_{j}(z)$ and $\mathbf{B}_{i}^{j}(z)$ in the parameter vector $\boldsymbol{\theta}_{v}^{j}$ leads to the following time-domain expression of $v_{d y n}^{j}(k)$ :

$$
v_{d y n}^{j}(k)=\left(\phi_{v}^{j}(k)\right)^{T} \boldsymbol{\theta}_{v}^{j}
$$

with

$$
\boldsymbol{\theta}_{v}^{j}=\left[\begin{array}{lllll}
\boldsymbol{\alpha}_{j} & \mid & \boldsymbol{\beta}_{1}^{j} & \ldots & \boldsymbol{\beta}_{m}^{j}
\end{array}\right]^{T}
$$

where

$$
\begin{aligned}
\boldsymbol{\alpha}_{j} & =\left[\begin{array}{lll}
-a_{1}^{j} & \ldots & -a_{p}^{j}
\end{array}\right] \in \mathbb{R}^{p} \\
\boldsymbol{\beta}_{i}^{j} & =\left[\begin{array}{lll}
\boldsymbol{\beta}_{i_{1}}^{j} & \ldots & \boldsymbol{\beta}_{i_{n}}^{j}
\end{array}\right], i=1 . . m \\
\boldsymbol{\beta}_{i_{l}}^{j} & =\left[\begin{array}{lll}
b_{1}^{j_{i l}} & \ldots & b_{\rho_{i}}^{j_{i l}}
\end{array}\right] \in \mathbb{R}^{\rho_{i}}, l=1 . . n
\end{aligned}
$$

so that $\boldsymbol{\theta}_{v}^{j} \in \mathbb{R}^{\nu}, \nu=p+n \sum_{i=1}^{m} \rho_{i}$.

The corresponding vector $\varphi_{v}^{j}(k)$ is:

$$
\phi_{v}^{j}=\left[\begin{array}{lllll}
\boldsymbol{\psi}_{j} & \mid & \boldsymbol{\varphi}_{1} & \ldots & \boldsymbol{\varphi}_{m}
\end{array}\right]^{T}
$$

with

$$
\begin{aligned}
\boldsymbol{\psi}_{j} & =\left[\begin{array}{lll}
v_{d y n}^{j}(k-1) & \ldots & v_{d y n}^{j}(k-p)
\end{array}\right] \in \mathbb{R}^{p} \\
\boldsymbol{\varphi}_{i} & =\left[\begin{array}{lll}
\boldsymbol{\varphi}_{i_{1}} & \ldots & \boldsymbol{\varphi}_{i_{n}}
\end{array}\right], i=1 . . m \\
\boldsymbol{\varphi}_{i_{l}} & =\left[\begin{array}{lll}
u_{i_{l}}(k-1) & \ldots & u_{i_{l}}\left(k-\rho_{i}\right)
\end{array}\right] \in \mathbb{R}^{\rho_{i}}, l=1 . . n
\end{aligned}
$$

\section{APPENDIX B}

Two-degrees of freedom example : Consider a robot with two joints $(n=2)$, and a detection scheme based on two input types $(m=2)$, namely $\mathbf{u}_{1}=\operatorname{sign}(\dot{\boldsymbol{\theta}})=$ $\left[\operatorname{sign}\left(\dot{\theta}_{1}\right) \operatorname{sign}\left(\dot{\theta}_{2}\right)\right]=\left[\begin{array}{ll}u_{11} & u_{12}\end{array}\right]$ and $\mathbf{u}_{2}=\ddot{\boldsymbol{\theta}}=$ $\left[\begin{array}{ll}\ddot{\theta}_{1} & \ddot{\theta}_{2}\end{array}\right]=\left[\begin{array}{ll}u_{21} & u_{22}\end{array}\right]$. A second order filter $\mathbf{G}$ is used $(\eta=2)$. In the dynamic threshold computations, denominators $A_{j}$ of degree $p=20$ are used, and numerators $B^{j}$ have degree $\rho_{1}=2$ for $\mathbf{u}_{1}$ and $\rho_{2}=4$ for $\mathbf{u}_{2}$. For the joint $j=1 . .2$, we have therefore:

$$
\begin{aligned}
v_{d y n}^{j}(k)= & \frac{1}{A_{j}(z)}\left[B_{11}^{j}(z) u_{11}(k)+B_{12}^{j}(z) u_{12}(k)\right. \\
& \left.+B_{21}^{j}(z) u_{21}(k)+B_{22}^{j}(z) u_{22}(k)\right]
\end{aligned}
$$

$B_{i_{l}}^{j}(z), i=1 . .2, l=1 . .2$ are polynomials in the discrete variable $z$. For the dynamic threshold, the linear regression is written as:

$$
v_{d y n}^{j}(k)=\left(\phi_{v}^{j}(k)\right)^{T} \boldsymbol{\theta}_{v}^{j}
$$

with

$$
\boldsymbol{\theta}_{v}^{j}=\left[\begin{array}{lllll}
\boldsymbol{\alpha}_{j} & \boldsymbol{\beta}_{11}^{j} & \boldsymbol{\beta}_{12}^{j} & \boldsymbol{\beta}_{21}^{j} & \boldsymbol{\beta}_{22}^{j}
\end{array}\right]^{T}
$$

where

$$
\begin{aligned}
& \boldsymbol{\alpha}_{j}=\left[\begin{array}{lll}
-a_{1}^{j} & \ldots & -a_{20}^{j}
\end{array}\right] \\
& \boldsymbol{\beta}_{11}^{j}=\left[\begin{array}{ll}
b_{1}^{j_{11}} & b_{2}^{j_{11}}
\end{array}\right] \\
& \boldsymbol{\beta}_{12}^{j}=\left[\begin{array}{ll}
b_{1}^{j_{12}} & b_{2}^{j_{12}}
\end{array}\right] \\
& \boldsymbol{\beta}_{21}^{j}=\left[\begin{array}{llll}
b_{1}^{j_{21}} & b_{2}^{j_{21}} & b_{3}^{j_{21}} & b_{4}^{j_{21}}
\end{array}\right] \\
& \boldsymbol{\beta}_{22}^{j}=\left[\begin{array}{llll}
b_{1}^{j_{22}} & b_{2}^{j_{22}} & b_{3}^{j_{21}} & b_{4}^{j_{21}}
\end{array}\right]
\end{aligned}
$$

so that $\theta_{v}^{j} \in \mathbb{R}^{\nu}, \nu=p+n \sum_{i=1}^{m} \rho_{i}$.

The corresponding vector $\varphi_{v}^{j}(k)$ is:

$$
\phi_{v}^{j}=\left[\begin{array}{lllll}
\boldsymbol{\psi}_{j} & \boldsymbol{\varphi}_{11} & \boldsymbol{\varphi}_{12} & \boldsymbol{\varphi}_{21} & \boldsymbol{\varphi}_{22}
\end{array}\right]^{T}
$$

with

$$
\begin{aligned}
\boldsymbol{\psi}_{j} & =\left[\begin{array}{lll}
v_{d y n}^{j}(k-1) & \ldots & v_{d y n}^{j}(k-20)
\end{array}\right] \\
\boldsymbol{\varphi}_{11} & =\left[\begin{array}{lll}
u_{11}(k-1) & u_{11}(k-2)
\end{array}\right] \\
\boldsymbol{\varphi}_{12} & =\left[\begin{array}{lll}
u_{12}(k-1) & u_{12}(k-2)
\end{array}\right] \\
\boldsymbol{\varphi}_{21} & =\left[\begin{array}{lll}
u_{21}(k-1) & \ldots & u_{21}(k-4)
\end{array}\right] \\
\boldsymbol{\varphi}_{22} & =\left[\begin{array}{lll}
u_{22}(k-1) & \ldots & u_{22}(k-4)
\end{array}\right]
\end{aligned}
$$

\section{REFERENCES}

[1] E. Datteri, G. Teti, C. Laschi, G. Tamburrini, G. Dario, and E. Guglielmelli, "Expected perception: an anticipation-based perceptionaction scheme in robots," in IEEE/RSJ International Conference on Intelligent Robots and Systems, vol. 1, 2003, pp. 934-939.

[2] D. Kulić and E. A. Croft, "Safe planning for human-robot interaction," Journal of Robotic Systems, vol. 22, no. 7, pp. 383-396, 2005.

[3] D. Kulić and E. Croft, "Pre-collision safety strategies for human-robot interaction," Autonomous Robots, vol. 22, no. 2, pp. 149-164, 2007.

[4] N. Najmaei and M. R. Kermani, "Prediction-based reactive control strategy for human-robot interactions," in IEEE International Conference on Robotics and Automation, 2010, pp. 3434-3439.

[5] C.-N. Cho, J.-H. Kim, Y.-L. Kim, J.-B. Song, and J.-H. Kyung, "Collision detection algorithm to distinguish between intended contact and unexpected collision," Advanced Robotics, vol. 26, no. 16, pp. 18251840, 2012.

[6] S. Haddadin, A. Albu-Schäffer, and G. Hirzinger, "Requirements for safe robots: measurements, analysis and new insights," The International Journal of Robotics Research, vol. 28, no. 11-12, pp. 1507-1527, 2009.

[7] IFA, "BG/BGIA risk assessment recommendations according to machinery directive, design of workplaces with collaborative robots. U 001/2009e (October 2009 edition, revised February 2011, future edition: Hazard Identification Recommendations of the Accident Insurers, EGU)," www.dguv.de/ifa/en/fac/kollaborierende_roboter/index.jsp, 2009.

[8] Y. Kishi, Y. Yamada, and K. Yokoyama, "The role of joint stiffness enhancing collision reaction performance of collaborative robot manipulators," in IEEE/RSJ International Conference on Intelligent Robots and Systems (IROS2012),, 2012, pp. 376-381. 
[9] Y. Yamada, Y. Hirasawa, S. Huang, Y. Umetani, and K. Suita, "Humanrobot contact in the safeguarding space," IEEE/ASME Transactions on Mechatronics, vol. 2, no. 4, pp. 230-236, 1997.

[10] B. Povse, D. Koritnik, R. Kamnik, T. Bajd, and M. Munih, "Emulation system for assessment of human-robot collision," Meccanica, vol. 46, no. 6, pp. 1363-1371, 2011.

[11] A. De Luca, A. Albu-Schaffer, S. Haddadin, and G. Hirzinger, "Collision detection and safe reaction with the DLR-III lightweight manipulator arm," in IEEE/RSJ International Conference on Intelligent Robots and Systems, 2006, pp. 1623-1630.

[12] S. Lu, J. H. Chung, and S. A. Velinsky, "Human-robot collision detection and identification based on wrist and base force/torque sensors," in IEEE International Conference on Robotics and Automation, 2005, pp. 3796 3801.

[13] G. Xiong, H. Chen, R. Zhang, and F. Liang, "Robot-environment interaction control of a flexible joint light weight robot manipulator," International Journal of Advanced Robotic Systems, vol. 9, 2012.

[14] M. Makarov, M. Grossard, P. Rodriguez-Ayerbe, and D. Dumur, "A frequency-domain approach for flexible-joint robot modeling and identification," in IFAC Symposium on System Identification, vol. 16, no. 1, 2012, pp. 583-588.

[15] H.-W. Je, J.-Y. Baek, and M. C. Lee, "Current based compliance control method for minimizing an impact force at collision of service robot arm," International Journal of Precision Engineering and Manufacturing, vol. 12, no. 2, pp. 251-258, 2011.

[16] R. Isermann, "Process fault detection based on modeling and estimation methods - a survey," Automatica, vol. 20, no. 4, pp. 387-404, 1984

[17] P. Frank and X. Ding, "Survey of robust residual generation and evaluation methods in observer-based fault detection systems," Journal of process control, vol. 7, no. 6, pp. 403-424, 1997.

[18] V. Venkatasubramanian, R. Rengaswamy, K. Yin, and S. N. Kavuri, "A review of process fault detection and diagnosis: Part I: Quantitative model-based methods," Computers \& Chemical Engineering, pp. 293 $311,2003$.

[19] S. Gadsden, Y. Song, and S. Habibi, "Novel model-based estimators for the purposes of fault detection and diagnosis," IEEE/ASME Transactions on Mechatronics, vol. 18, no. 4, pp. 1237-1249, 2013.

[20] H. Schneider and P. Frank, "Observer-based supervision and fault detection in robots using nonlinear and fuzzy logic residual evaluation," IEEE Transactions on Control Systems Technology, vol. 4, no. 3, pp. 274-282, 1996.

[21] S. Haddadin, A. Albu-Schaffer, A. De Luca, and G. Hirzinger, "Collision detection and reaction: A contribution to safe physical human-robo interaction," in IEEE/RSJ International Conference on Intelligent Robots and Systems, 2008, pp. 3356-3363.

[22] W. Dixon, I. Walker, D. Dawson, and J. Hartranft, "Fault detection for robot manipulators with parametric uncertainty: a prediction-error-based approach," IEEE Transactions on Robotics and Automation, vol. 16, no. 6, pp. 689-699, 2000.

[23] C.-N. Cho and J.-B. Song, "Collision detection algorithm robust to model uncertainty," International Journal of Control, Automation and Systems, vol. 11, no. 4, pp. 776-781, 2013.

[24] S. Morinaga and K. Kosuge, "Collision detection system for manipulator based on adaptive impedance control law," in IEEE International Conference on Robotics and Automation, vol. 1, 2003, pp. 1080-1085.

[25] A. De Luca and R. Mattone, "An adapt-and-detect actuator FDI scheme for robot manipulators," in IEEE International Conference on Robotics and Automation, vol. 5, 2004, pp. 4975-4980.

[26] A. De Luca and W. Book, "Robots with flexible elements," in Springer Handbook of Robotics, B. Siciliano and O. Khatib, Eds. Springer, 2008, pp. 287-319.

[27] M. Makarov and G. M, "Modeling and motion control of serial robots with flexible joints," in Flexible Robotics: Applications to Multiscale Manipulations, M. Grossard, N. Chaillet, and S. Régnier, Eds. Wiley, 2013.

[28] A. Caldas, M. Makarov, M. Grossard, P. Rodriguez-Ayerbe, and D. Dumur, "Adaptive residual filtering for safe human-robot collision detection under modeling uncertainties," in IEEE/ASME International Conference on Advanced Intelligent Mechatronics, 2013, pp. 722-727. 